\title{
THE COGNITIVE-BEHAVIORAL THERAPY IN THE TREATMENT OF PATIENTS SUFFERING FROM KINESIOPHOBIA
}

\author{
Marta GÓRSKA \\ Military Institute of Aviation Medicine, Department of Aviation Psychology, Warsaw, Poland
}

Source of support: Own sources

Author's address: M. Górska, Institute of Aviation Medicine, Krasińskiego 54/56 Street, 01-755 Warsaw, Poland, e-mail: marrta.gorska@gmail.com

Abstract: The aim of the article is to present a case study and the role of inclusion of cognitivebehavioral psychotherapy in the physiotherapy of patients suffering from kinesiophobia. This disorder is characterized by a strong fear of physical activity, exercise and activation after surgery for fear of injury. It occurs most frequently after orthopaedic surgeries, and the most common reasons for treatment in this regard are lesions of traumatic or degenerative nature, as well as the presence of the chronic pain syndrome. Kinesiophobia is a psychological risk factor, which is important for the progress of physiotherapeutic treatment, as its presence can significantly slow down the recovery process. Early detection of the fear of movement and the observation that the patient avoids behavioral activity may have a positive impact on the patient's involvement in postoperative treatment and faster recovery.

The inclusion of cognitive-behavioral therapy techniques in patients with mobility anxiety and long-term pain can significantly affect the rate of recovery.

Keywords: cognitive-behavioral psychotherapy, kinesiophobia, anxiety disorders, physiotherapy, psychosomatics, chronic pain

Figures: 2 - Tables: 2 - References: 19 - Full-text PDF: http://www.pjambp.com • Copyright @ 2016 Polish Aviation Medicine Society, ul. Krasińskiego 54/56, 01-755 Warsaw, license WIML •Indexation: Index Copernicus, Polish Ministry of Science and Higher Education 


\section{INTRODUCTION}

The identification of psychological risk factors, and in particular the presence of kinesiophobia in the patient, makes it possible to plan a physiotherapy plan and a specific approach of medical personnel to the patient, as well as to estimate the outcome of the postoperative procedure and the course of rehabilitation in the context of the success of physiotherapy. Many of the results of the study focused on distinguishing psychological risk factors, the level of chronic pain sensation due to the structure of the patient's personality, which may disturb and disturb the pace of recovery $[1,2]$. It was emphasized that the dominant psychological risk factors that constitute a barrier to the success of treatment may be the nonconstructive strategies of coping with pain, the psychological distress experienced, diagnosed psychological disorders, the tendency to experience strong emotions manifested in the patient's body (non-specific pains), fear of movement and bodily injury (kinesiophobia), as well as beliefs, pain behaviors (beliefs concerning pain, expectations about treatment, interpretation of one's situation in pain), approach to work (interpretation of work as something tedious, burdensome). Next, we can point to the risk factor, which is characterized by the patient's tendency to catastrophize, which manifests itself in the thought process of a patient suffering from chronic pain, e. g."pain is terrible, it will get worse and worse","my suffering will never end". Research results indicate that physical factors are not always the source of chronic pain. Special attention is paid to patients who, in imaging and physical examination, do not show evidence of sensed pain symptoms (particularly migratory, appearing in various parts of the body), or neurological symptoms such as numbness and tingling of the limbs, stiffening of the limbs, loss of local sensation. For this reason, the clinicians decided to evaluate the importance of psychological factors in sensing pain as well as other somatic symptoms and put them in the diagnostic process in the form of yellow flags. Physiotherapy flags are a marker of risk factors for musculoskeletal disorders. The yellow flag indicates psychosocial factors (depression, anxiety, etc.) as well as the patient's beliefs about their condition. The evaluation of psychological factors through yellow flags focuses primarily on what the patient thinks about the treatment and pain, the attitudes and strategies of dealing with pain. Another aspect is what they expect of the treatment and whether they make claims in their own health situation, as well as the manner in which the patient understands the diagnosis and how they perceive the manner in which they receive information on their health status. Emotional problems and the level of emotions, especially the degree of severity of depression and anxiety, which are important for the intensity of pain, the family's reaction to the patient's state of health and whether the patient has support from relatives, also play an important role. Attention is also drawn to the professional situation and the relationship between the patient and their return to work, how the patient evaluates their job. Research on the effectiveness of cognitive-behavioral psychotherapy in the treatment of patients with chronic pain and kinesiophobia yields satisfactory results. Participation in cognitive behavioral psychotherapy and the use of cognitive-behavioral psychotherapy means that patients perceive pain as less severe, also indicating better quality of life compared to pre-treatment time. Moreover, patients who did not participate in cognitive-behavioral therapy were less able to control pain and showed more negative thoughts and emotions in relation to the interpretation of disability [3].

\section{FEAR AVOIDANCE MODEL}

Model Unikania Strachu or Model Unikania Lękowego are actually polish names for the Fear Avoidance Model, which are using interchangeably. This model was introduced by Lethem in 1983 and helped to explain how people feel pain in spite of no physicalpathology. Figure 1 concerns experiencing pain in two ways. On the one hand, if a person experiences the painful discomfort associated with bodily injury and takes an avoiding stance, then even if the pain is not intense, the degree of intensity of emotions experienced in the form of anxiety, above all, will increase the feeling of somatic ailments $[4,5]$. As can be seen in the diagram, the sensitivity of perceiving pain signals is increased, which confirms that something dangerous happens to the patient's body and they takes the stance of avoiding behaviors associated with experiencing pain $[6,7]$. On the other hand, if a person feels pain and interprets it as harmless or temporary, they feel less anxiety and faces the painful situation head on. Avoidance in itself as a way of dealing with pain has its advantages and disadvantages. It can be said that avoiding pain is healthy if the patient rationally evaluates what is harmful to them and what is not. In spite of the pain, the patient will adapt to the physiotherapist's recommendations, they will try to cope with 
disability adequately to their health condition, and will display both internal and external sense of responsibility for their health. A patient who interprets pain as threatening shows excessive vigilance and concentration on pain symptoms and disability limits their treatment and thus does not utilize their physical resources by delaying treatment, which is not beneficial in physical or mental terms.

According to the author of the article, the above model can be applied not only for the development and maintenance of chronic pain, but also for kinesiophobia. The presence of kinesiophobia can be taken as a psychological risk factor in the context of recovery. This term is understood as the fear of movement and physical activity resulting from the feeling of vulnerability to injury or damage (Kori et. all, 1990), but also as the attitude towards activity and the manifestation of personal disposition [8]. According to the author, the Fear Avoidance Model indicates that when experiencing pain is interpreted as threatening and is cognitively distorted by the patient, and in this case catastrophization is apparent ("I can't take it","it will never end","the surgery failed"), as a result, the patient, through increased muscular tension, evaluates the experienced pain as more intense and begins to interpret it in terms of a threat to their body (damage). The intensity of experiencing pain is influenced by the psychological distress experienced at the moment of pain. The experienced emotions of anxiety and depression amplify the pain signals, as Melzack and Wall wrote in 1965, creating the Gate Control Theory of Pain. It assumes that anxiety, depression, anxiety and other psychological factors that have been identified as "reactions to pain" have begun to be considered as an integral part of the processing of pain-related information [9]. The input signal for experiencing pain is the presence of a stimulus, which is the input for experiencing pain. The signal is transmitted along the spinal cord and then interpreted in the brain, resulting in experiencing pain. The Gate Control theory assumes that at the stage of signal transmission to the spinal cord, a control gate mechanism appears, which is responsible for the selection of signals that will be sent for further interpretation in the brain. Depending on the "opening width" of the gate, we will perceive the pain as more or less intense. Experiencing pain is a resultant of physical and mental factors. The opening or closing of the gate will depend on the occurrence of a physical factor (e. g. an injury), but the role of psychological factors should not be downplayed here. When the unpleasant emotions experienced manifest themselves in a somatic form, we can perceive them in the form of increased blood pressure, accelerated heart rate, increased muscle tension, as well as increased hormonal secretion. The above physiological condition will have an impact on the amplification of the perceived pain signals, the greater the pain, the worse the emotional condition will be, and this in turn will intensify the pain. The second psychological mechanism of processing pain stimuli is responsible for the opening width of the pain gateway, and thus the intensity of the permeable signal, and it is at this point that the experienced emotions can have an impact on the amplification of the received signal, which affects the severity of the pain [10].

According to the Fear Avoidance Model and nonconstructive coping with pain, the interpretation of a pain experience leads to avoidance of activity and behaviors related to rehabilitation and health. The patient does not want to exercise, does not want to walk, does not see themselves as a professionally active person, which may be the result of catastrophic thinking and severe anxiety. Generally speaking, one can say that they start to close themselves in the "pain bubble" and a completely new way of thinking about themselves, as a person who is not fully functional, while experiencing a lot of unpleasant emotions in the form of anxiety, sadness, grief and guilt. It is apparent that they separate themselves from the outside world, people, friends and acquaintances, and go on living in an increasingly strong cognitive distortion concerning the future, perceiving it in black-andwhite colors and an excessively negative light [11].

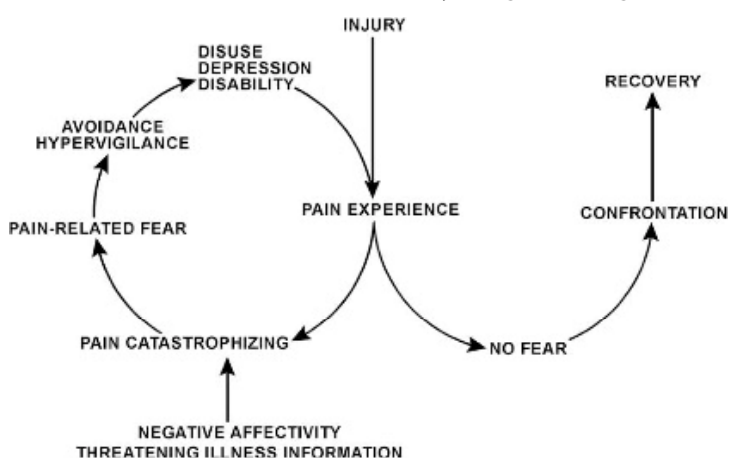

Fig. 1. Fear Avoidance Model (source: http://www. physio-pedia.com/File:Pain_fear. jpg).

Recognition of psychological risk factors and, above all, early diagnosis of kinesiophobia is extremely important when planning postoperative treatment. Many studies have shown that early diagnosis of psychological risk factors can prevent adverse treatment outcomes [12,13]. Scientific data and clinical observations indicate that, in addition 
to an excessive concentration on pain sensations, the diagnosis of kinesiophobia as erroneous and inadequate assessment of pain and anxiety concerning the re-occurrence of body injuries is relevant to the etiology of chronic pain and related disability. These discoveries are consistent with the cognitive-behavioral perspective, which emphasizes the importance of maladjusted somatic interpretations. This mechanism can be described as follows: people who misinterpret bodily feelings, including pain, in a catastrophic and disastrous manner may experience anxiety regarding pain, resulting in at least two processes. First of all, anxiety associated with pain is related to avoidance behaviors, especially avoidance of movement and physical activity. Avoidance is not only the avoidance of physiotherapy activity, but also avoidance of work, social encounters and other behavioral activities. Secondly, the anxiety associated with pain is associated with increased carnal consciousness and hypersensitivity to pain. Excessive vigilance, depression and inactivity are associated with increased pain levels, and can thus exacerbate painful experiences [14].

\section{COGNITIVE-BEHAVIORAL THERAPY IN THE TREATMENT OF PATIENTS SUFFERING FROM KINESIOPHOBIA}

The presence of anxiety concerning movement in the patient predisposes them to obtaining undesirable postoperative results. Among the four main trends in psychotherapy, there is the cognitive-behavioral approach, which is characterized by an exceptionally good level of effectiveness in the treatment of patients with diagnosed kinesiophobia. Cognitive-behavioral therapy (CBT) has strong empirical support, particularly visible is the efficacy of cognitive and behavioral techniques when working with patients with anxiety disorders, including kinesiophobia $[15,16]$. Interventions in cognitive behavioral therapy include supporting time management, problem solving, cognitive restructuring, careful learning, respiratory techniques and relaxation strategies.

An example of the efficacy of the cognitive-behavioral approach can be found in a study aimed at determining the effects of the cognitive-behavioral approach implemented alongside physiotherapy in patients with high levels of movement anxiety. The study group consisted of patients with degenerative lesions of the lumbar segment qualified for lower spinal section surgery. The aim of the CBT techniques was to influence the fear of motion through e.g. psycho-education, teaching self-management and techniques of cognitive restructuring aimed at catastrophic thinking in order to increase physical activity. The study involved 130 patients randomly assigned to a programme consisting of physical exercise and cognitivebehavioral therapy in an experimental group (65 people) or physical exercise only (control group, 65 people). Before treatment, 4 weeks later and 12 months after termination of treatment (followup). All patients completed questionnaires on the degree of disability, assessing the severity of kinesiophobia. The results of this study indicate that the rehabilitation programme combined with the use of cognitive-behavioral techniques was better than the programme without the implementation of psychotherapeutic techniques, taking into account the severity of catastrophic thoughts and the assessment of the quality of life by the patients.

Despite the fact that the perception of oneself as a person with disabilities appeared in both groups, the experimental group patients assessed health improvement as higher. It turned out that, after an instructor given to patients on how to control and modify erroneous, catastrophic beliefs and anxiety thoughts, as well as encouraging them to take appropriate actions towards perceiving disability, it reinforced their positive attitude towards exercise and increased their physical fitness. Patients were eager to undertake exercises in accordance with previous recommendations [16]. In terms of the presence of the fear of movement and the level of catastrophization, there was a significant difference between the control and the experimental group. In the experimental group, the above mentioned components were minimized, while in the control group their level remained unchanged throughout the whole study period, thus constituting the main barrier to improvement of physical fitness and general health [17].

The results obtained in the quality of life monitoring scale suggested tangible benefits in gaining control over thinking and coping with illness. The improvement was always much greater in the experimental group.

Christensen also points to the positive role of the psychotherapeutic approach applied simultaneously with physiotherapy. This is particularly true for patients suffering from prolonged pain. The combined cognitive-behavioral and physiotherapy programme is usually applied 3 months after surgery, and this time is necessary to avoid 
overloading the stabilization of the lower spine lumbar region. Abbitt's results indicate that interventions associated with cognitive-behavioral therapy are fully safe and have a long-term effect on the management of pain and severity of kinesiophobia after surgery. The cooperation between the physiotherapist and psychotherapist can contribute to a faster recovery of the patient. During physiotherapy, the presence of a psychotherapist is very important, for example, in order to intervene quickly in the case of cognitive and behavioral difficulties that a physiotherapist will notice $[18,19]$.

In addition, psychotherapeutic measures are primarily aimed at controlling catastrophic thinking, interpreting pain experiences and inappropriate behaviors, and developing relaxation techniques and cognitive strategies for dealing with stress instead.

The rehabilitation programme, which includes CBT elements, including self-management and exposure to mobility anxiety, is useful for achieving the desired treatment outcomes, the degree of intensity of catastrophic thinking, beliefs about pain and a general assessment of quality of life.

\section{DESCRIPTION OF THE CASE}

The patient aged 37 years reported for psychotherapy at their physiotherapist's recommendation. The psychotherapy was conducted in the cognitive-behavioral field by the author of the article. There were 12 individual weekly psychotherapeutic sessions.

The patient was involved in a motorcycle accident, which resulted in a serious knee joint injury in the form of rupture of the cruciate ligaments of the knee joint. Breaking the anterior ligament is one of the most common injuries of the knee joint. Depending on the injury mechanism, other joint structures may also be damaged, as in the case of the O'Donoghue triad, the tibial collateral ligament and the falciform cartilage are additionally damaged. There are as many as eleven ligaments in the knee joint, which stabilize and enable its proper functioning. The anterior cruciate ligament is one of the most important ligaments in the joint and prevents the tibia from sliding forward in relation to the femur. Patients who did not undergo reconstruction treatment experience more rapid appearance of degenerative lesions. The patient, 9 months before the first therapeutic meeting, went through the reconstruction of the anterior cruciate ligament with the internal bracing method and reconstruction of the secondary tibial ligament. The rehabilitation consisted of 2 parts. The first involved preparing the patient for the procedure, and the second to restore full mobility after the procedure. After the surgery, physiotherapy began with weekly meetings with a physiotherapist, three times a week. Rehabilitation was very slow, the patient used her the lower limb with great caution. She was afraid to stand on the operated leg alone despite the fact that it was not a physical danger to her. She stepped with great care, observed them all the time and paid great attention to the correctness of walking. The patient, despite a good operating result confirmed by the imaging studies, indicated that she was permanently suffering from pain with a high intensity. VAS measurement amounted to 8 (tab. 1). The VAS scale is a tool that is used when assessing the severity of pain sensations. The patient evaluates pain on a scale from 0 - complete painlessness to 10 - the strongest imaginable pain and, based on their own sensations and references to other pain experiences, they evaluate the severity of pain at a given moment in time. The patient indicated that it was particularly difficult for her to walk on uneven and wet ground. As a result, her social and professional activity has been significantly reduced. Before the walk, the patient planned exactly the route of the walk - she asked the relatives to check whether the pavement surface was certainly even on the planned road. She made sure that the weather conditions would not change and that the rain or snow would not fall at a given time, which could endanger stability. The patient's feelings may more accurately be reflected by the content of the message about coping with disability. The description of feelings was an element of the patient's homework as part of psychotherapy: "On Thursday I walked home carefully, there were barely the first snowflakes, nonetheless, it was wet, so I walked with caution, phew. It was not easy on Friday, but I decided to face the winter. I walked cautiously, I imagined walking on berets, it is also a non-stable surface. Slowly, looking calmly at my feet, although somewhere in the back of my head I heard Tomek's voice "Kasia, look where you're going, nothing is happening underneath my feet". And at this point a slight slip/instability first affected the healthy leg (right) and then the sick leg (left). Instantaneously my muscles became tight. I stopped and calmly worked out the whole situation in my head and that nothing happened to me, and calmly, slowly and without hurry I will reach my destination, never mind the snow, it's going to thaw anyway (yes, I know, I exaggerated a little with this thawing business and I shouldn't have to shortened it so much, 
but the destination was already on the horizon). I got to the bakery, but I had to climb the stairs, so I got my feet together, but I had to go down too. I didn't walk down the stairs that I had walked in on, I took the ones where people were, so I could grab someone at any time or simply ask for help. But I also walked down alone, step by step, without any help. Lucky, I took a car for this walk. And so, going towards the above, I was caught by an older man with the question: "Please be careful! Do you need help? Did something happen? I thanked him politely with a smile and I walked very cautiously on the snow puddle looking for a place without snow and its thawing remains. Of course, the idea that there should be fines for snow-covered pavements repeatedly passed through my head, but it was more important to get to the bakery first and then to the car safely. I had to continue this walk later in another place in more beautiful surroundings, but having walked about 500 m exhausted me".

Psychotherapeutic meetings started with a psychological diagnosis aimed at looking at psychological factors that could have an impact on the pace of the treatment process. Attention was drawn to such factors as: the general level of experienced psychological distress, the strategy of coping with pain and the location of pain control. On the basis of the obtained data and personality profile as well as the interview data, it was possible to conclude about the existence of features characteristic of an obsessive-compulsive personality, however not meeting the full diagnostic criteria to make an unambiguous diagnosis. The patient presented herself as task-oriented and striving for perfection. The respondent showed the need to dominate, to strive for the best achievements, did not give herself the right to make mistakes.

The action style based on emotions was very weak and the main defensive mechanism was denial of psychological problems. The degree of pain intensity is shown in Table 1. The patient was asked to respond on a 10 degree scale to the question of how much pain she felt. The VAS scale mentioned above was used for this measurement.

Tab. 1. Subjective assessment of the patient's pain measured with the VAS scale (measurement before cognitive-behavioral therapy).

VAS (VISUAL ANALOG SCALE)

$\begin{array}{llllllllllll}1 & 2 & 3 & 4 & 5 & 6 & 7 & 8 & 9 & 10\end{array}$

The patient assessed the degree of pain at eight on a ten-degree scale. This is a high result. The fear of movement was measured. The measurement was performed with the use of the psychotherapist's own scale (author of the article) measuring the degree of kinesiophobia intensity. The scale is currently being validated. The maximum score of the scale is 68 points. The patient scored 59 points, which may indicate a high level of fear of moving.

12 therapeutic sessions were conducted in order to minimize the fear of movement. Individual psychotherapeutic sessions were initially aimed at getting to know the patient and creating a conceptualization of the problem of fear of movement. Thinking errors and cognitive distortions were indicated and the ways in which the patient interprets herself as not fully functional were traced. The ideas and thoughts that appeared during physiotherapy were examined. Around 5th session, a cognitive work started based on Socratic dialog technique and focusing on the patient's key beliefs. Around 7th session, behavioral psychotherapeutic intervention was included in the physiotherapeutic process. The experiment was planned which the patient was to carry out under the care of a physiotherapist each time during the visit. When anxiety thoughts appeared, she was to use alternative thoughts. The chart below shows that the fear of moving decreased when the patient engaged in the experiment, which was also visible in the dynamics of the patient's movement during physiotherapy.

After the psychotherapeutic treatment, the intensity of pain was re-measured (fig. 2). The patient evaluated her pain using the VAS scale, on which she had previously estimated her pain at 8/10 (tab. 1).

After applying psychotherapeutic impacts simultaneously with physiotherapy, the patient evaluated the pain at 5/10 (tab. 2).

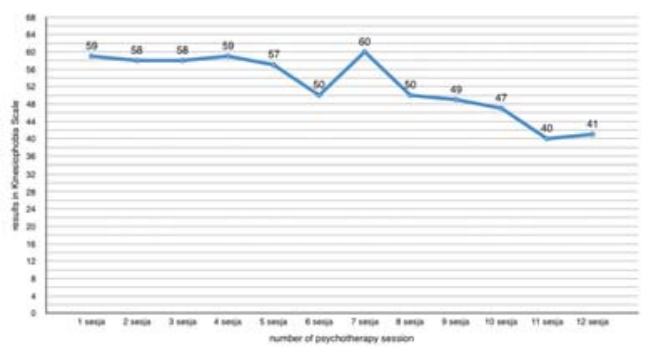

Fig. 2. Changes in the intensity of fear of movement in the physiotherapeutic and psychotherapeutic approach implemented simultaneously.

Tab. 2. Subjective assessment of the patient's pain measured with the VAS scale (measurement after cognitive-behavioral therapy).

VAS (VISUAL ANALOG SCALE)

\begin{tabular}{llll|l|lllll}
1 & 2 & 3 & 4 & 5 & 6 & 7 & 8 & 9 & 10
\end{tabular}


There is not enough data to state unequivocally that the patient's participation in psychotherapy has reduced the severity of pain, however, a hypothesis may be assumed that participation in psychotherapeutic meetings was significant for the experiencing emotional and physical tensions. However, the above case description requires a thorough analysis performed on an appropriately large group of respondents.

\section{CONCLUSIONS}

A review of research as well as own experience may suggest that monitoring of psychological risk factors in the context of coping in postoperative and physiotherapy period is important to achieve the best treatment outcomes. Psychotherapeutic procedures implemented in parallel with physiotherapy may provide support not only for the patient, but also for the medical personnel - physiotherapists, physicians, as well as administration staff. The treatment process in some patients is long and monotonous, it can be seen that the patient manifests resistance in contact, is insecure and gives the impression of having no motivation to recover. This may be a good time to direct the patient to a psychotherapist. The situation of dis- ability and illness itself is a huge disorder of homeostasis in the patient's everyday life. The patients must find themselves in a new role and reorganize many aspects of their life. Therefore, they may experience many unpleasant emotions such as depression, anxiety, anger, which are important for muscle tension and thus for the progress of treatment.

Determination of psychological risk factors before surgery may be important information for the attending physician, but above all for the patient. Recognition of psychological risk factors that may block and inhibit the progress of treatment may be important information necessary in the preparation for the medical procedure. Systematizing the role of psychotherapy in pre- and postoperative treatment and including it in the standards of preparation of the patient for the surgery would allow minimizing the risk of long recovery from surgery. Further studying of the impact of psychological risk factors, the structure of a patient's personality and typical patient's response patterns may provoke the discovery of new, interdisciplinary treatment methods and thus lead to better medical interventions affecting the quality of patients' life.

\section{AUTHORS' DECLARATION:}

Study Design: Marta Górska; Data Collection: Marta Górska; Manuscript Preparation: Marta Górska; Funds Collection: Marta Górska. The Author declares that there is no conflict of interest.

\section{REFERENCES}

1. Glomsrød, Bredo; Lønn, Jan Harald \& Vøllestad, Nina Køpke (2007). Prognostic factors in first-time care seekers due to acute low back pain. European Journal of Pain. ISSN 1090-3801. 11(3): 290-298.

2. Brox JI1, Storheim K, Holm I, Friis A, Reikerås O. Disability, pain, psychological factors and physical performance in healthy controls, patients with sub-acute and chronic low back pain: a case-control study.

3. McCracken, Lance M. PhD; Turk, Dennis C. PhD Behavioral and Cognitive-Behavioral Treatment for Chronic Pain: Outcome, Predictors of Outcome, and Treatment Process, Spine: November 15th, 2002 - Volume 27 - Issue 22 - p 2564-2573.

4. Herbert H. Zaretsky; Edwin F. Richter; Myron G. Eisenberg (21 June 2005). Medical Aspects Of Disability: A Handbook For The Rehabilitation Professional. Springer Publishing Company. pp. 223-4. ISBN 978-0-8261-7973-9. Retrieved 19 July 2012.

5. Asmundson, Gordon; Norton, Peter (1999). „Beyond pain: the role of fear and avoidance in chronicity” (PDF). Clinical Psychology Review. 19 (1):97-119. doi:10.1016/S0272-7358(98)00034-8.

6. Pincus, Tamar; Smeets, Rob J.E.M.; Simmonds, Maureen J.; Sullivan, Michael J.L. (November 2010). „The Fear Avoidance Model Disentangled: Improving the Clinical Utility of the Fear Avoidance Model”. The Clinical Journal of Pain. 26 (9):739-746.

7. Crombez, Geert; Eccleston, Christopher; Van Damme, Stefaan; Vlaeyen, Johan W. S.; Karoly, Paul (Jul 2012). „Fear-avoidance model of chronic pain: the next generation". The Clinical Journal of Pain. 28(6):475-483.

8. A Knapik, E Saulicz, R Gnat Kinesiophobia-introducing a new diagnostic toolJournal of human kinetics 28, 25-31 
9. Melzack R, Wall PD. Pain mechanisms: A new theory. Science. 1965; 150:971-9.

10. Melzack R, Wall PD. Citation Classic - Pain Mechanisms: A new theory. Current Contents. 1982; 22.

11. Vlaeyen JW, Kole Snijders AM, Rotteveel AM, Ruesink R, Heuts PH. The role of fear of movement (re)injury in pain disability. J Occup Rehabil. 1995;5(4):235-52.

12. Leeuw M, Houben RM, Severeijns R, Picavet HS, Schouten EG, Vlaeyen JW. Pain-related fear in low back paina prospective study in the general population. Eur J Pain. 2007;11(3):256-266.

13. Boersma K, Linton SJ. Psychological processes underlying the development of a chronic pain problema prospective study of the relationship between profiles of psychological variables in the fear-avoidance model and disability. Clin J Pain. 2006;22(2):160-166.

14. 13.Smeets RJ1, Vlaeyen JW, Kester AD, Knottnerus JA. Reduction of pain catastrophizing mediates the outcome of both physical and cognitive-behavioral treatment in chronic low back pain. J Pain. 2006 Apr;7(4):261-71.

15. Van Tulder MW, Ostelo RW, Vlaeyen JWS, et al. Behavioral treatment for chronic low back pain. A systematic review within the framework of the Cochrane Back Review Group. Spine 2001; 26:270-81.

16. Morley S. Efficacy and effectiveness of cognitive behaviour therapy for chronic pain: progress and some challenges. Pain. 2011;152:S99-S106. doi: 10.1016/j.pain.2010.10.042.

17. Crombez G, Vlaeyen JWS, Heuts PHTG, Lysens R. Fear of pain is more disabling than pain itself. Evidence on the role of pain-related fear in chronic back pain disability. Pain. 1999; 80:329-340. doi: 10.1016/S0304-3959(98)00229-2.

18. Butler G. Cognitive-Behavioral Therapy for Anxiety Disorders: Mastering Clinical Challenges (Guides to Individualized Evidence-Based Treatment).

19. Kristin R. Archer,corresponding author Nicole Motzny, Christine M. Abraham, Donna Yaffe, Caryn L. Seebach, Clinton J. Devin, Dan M. Spengler, Matthew J. McGirt, Oran S. Aaronson, Joseph S. Cheng, Stephen T. Wegener, Cognitive-Behavioral-Based Physical Therapy to Improve Surgical Spine Outcomes: A Case Series.

\section{ACKNOWLEDGEMENTS}

The views, opinions, and findings contained in this article are our own and should not be construed as an official Polish Air Force position, policy, or decision, unless so designated by other official documentation.

Cite this article as: Górksa M. The Cognitive-Behavioral Therapy in The Treatment of Patients Suffering from Kinesiophobia. Pol J Aviat Med Bioeng Psychol 2016; 22(4): 43-50. DOI: 10.13174/pjambp.23.02.2018.04 